\title{
Polymyxin B use Associated with Severe Hypotensive Episodes
}

\section{Mehta $\mathbf{M}^{1^{*}}$, Baron $\mathrm{JM}^{1}$, Nelson BC ${ }^{1}$, Muir $\mathrm{J}^{\mathbf{1}}$ and Pereira MR ${ }^{2}$}

${ }^{1}$ NewYork-Presbyterian Hospital, USA

${ }^{2}$ Columbia University Medical Center, USA

\begin{abstract}
Polymyxin B was developed in the 1940s but was infrequently used because of renal toxicity. Since the rise of infections due to multidrug resistant gram-negative organisms, polymyxin B has re-emerged as an important agent. However, its toxicity is still not fully elucidated. In this report, we describe two cases of multiple hypotensive events occurring after polymyxin B administration. Management strategies, such as slowing the infusion rate and administering diphenhydramine, did not mitigate the hypotension. We also describe relatively high polymyxin levels correlated with this effect in one of these cases.
\end{abstract}

\section{Keywords: Polymyxin; Toxicity; Hypotension}

\section{Case 1}

A 35-year-old-woman was admitted to our hospital for management of newly diagnosed acute promyelocytic leukemia. Twelve days after initiation of chemotherapy, she developed a fever to $38.0^{\circ} \mathrm{C}$. Cultures of her urine and blood grew a carbapenem-resistant Escherichia coli susceptible to polymyxin B (minimum inhibitory concentration $[\mathrm{MIC}]=2 \mathrm{mg} / \mathrm{L})$, amikacin $(\mathrm{MIC} \leq 2 \mathrm{mg} / \mathrm{L})$, and tigecycline $(\mathrm{MIC} \leq$ $0.5 \mathrm{mg} / \mathrm{L})$.

She was initiated on polymyxin B $300 \mathrm{mg}(3 \mathrm{mg} / \mathrm{kg})$ intravenously (IV) in $100 \mathrm{ml}$ over one-hour in addition to meropenem. After the first polymyxin $B$ dose, the patient experienced parasthesias in her face, arms, and trunk. These side effects prompted the polymyxin B to be discontinued and replaced by amikacin and meropenem. Due to continued concerns about her carbapenem-resistant $E$. coli infection, polymyxin B was re-initiated five days later with a maintenance dose of $150 \mathrm{mg}(1.5 \mathrm{mg} / \mathrm{kg})$ IV over one-hour every 12 hours along with meropenem. After the fourth dose, she experienced a syncopal event in which her blood pressure (BP) decreased from 112/79 prior to polymyxin $\mathrm{B}$ to $67 / 57 \mathrm{mmHg}$ thirty minutes after the infusion. Her BP increased to $90 / 50 \mathrm{~s}$ after IV fluids. She remained stable until after the 10th polymyxin B dose, when her BP decreased from 109/78 $\mathrm{mmHg}$ prior to polymyxin $\mathrm{B}$ to $89 / 69 \mathrm{mmHg}$ at the completion of the one-hour infusion. She also had tachycardia $(\mathrm{HR}=200)$ and altered mental status with complaints of dizziness, shortness of breath, and mouth/tongue tingling. In attempt to manage the adverse reactions, the next dose was reduced to $140 \mathrm{mg}$, infused over four hours, and administered after a dose of diphenhydramine $25 \mathrm{mg}$. In spite of this, after $75 \%$ of completion of the subsequent dose, her BP decreased once again from 117/75 mmHg prior to polymyxin $\mathrm{B}$ to $92 / 71 \mathrm{mmHg}$ during the infusion along with accompanying lethargy and neuropathic symptoms. The patient was then transferred to the intensive care unit for closer monitoring. During the 18th dose, the BP dropped again from $100 / 60 \mathrm{mmHg}$ prior to polymyxin $\mathrm{B}$ to $81 / 69 \mathrm{mmHg}$ during the infusion and her pressure increased spontaneously to $100 / 70 \mathrm{mmHg}$ after one hour. Polymyxin B therapy was completed after 14 days. She remained in the hospital for the next 17 days to complete her chemotherapy, during which she had intermittent episodes of hypotension. Polymyxin B serum levels were measured at steady state after three days of intravenous infusion. The polymyxin B peak and trough, combining both B1 and B2 forms, were $9.6 \mathrm{mg} / \mathrm{L}$ and $5.0 \mathrm{mg} / \mathrm{L}$, respectively. The estimated area under the curve at 24 hours (AUC24) was $172 \mathrm{mg}^{\star} \mathrm{h} / \mathrm{L}$.
While hypotension is usually a complication of sepsis, details in this case suggest that the episodes of hypotension are more related to polymyxin B administration than an uncontrolled infection. The episodes of hypotension started to occur only after polymyxin B and other antibiotics were initiated. There is good evidence, with negative follow-up cultures, resolution of fever, and improving imaging, that the infection was controlled by the time that the hypotensive episodes started. Furthermore, during the hypotensive episodes, the pre- and post-polymyxin B blood pressures were markedly different.

\section{Case 2}

A 23-year-old-man was admitted to our hospital with fever and upper respiratory tract infection symptoms which rapidly progressed to severe hypoxemic respiratory failure and acute respiratory distress syndrome (ARDS). As a salvage strategy for his hypoxemia, he was placed on extracorporeal membrane oxygenation (ECMO) and transferred to our medical intensive care unit (ICU). He was diagnosed with community acquired pneumonia and a respiratory polymerase chain reaction (PCR) swab was positive for Chlamydophila pneumoniae. Given the severity of his illness and vasopressor-dependent shock, he was initially treated with broad-spectrum antibiotics including vancomycin, piperacillin/tazobactam, and levofloxacin. Over the next several days, his shock resolved and he was weaned off vasopressors, but he had persistent fevers, rigors, and hypoxemia, and intermittently required neuromuscular blockade for ventilator dyssynchrony, so his antibiotics were escalated to vancomycin, meropenem, doxycycline, and tobramycin. Other than his initial positive respiratory PCR, all bacterial cultures remained negative.

On day 10 of admission, he unexpectedly suffered a cardiac arrest with pulseless electrical activity and achieved return of spontaneous circulation after approximately two minutes of cardiopulmonary

${ }^{*}$ Corresponding author: Monica Mehta, Pharm.D, M.P.H, New York-Presbyterian Hospital, USA, E-mail: mem9059@nyp.org

Received November 09, 2015; Accepted December 05, 2015; Published December 14, 2015

Citation: Mehta M, Baron JM, Nelson BC, Muir J, Pereira MR (2015) Polymyxin B use Associated with Severe Hypotensive Episodes. J Antimicro 1: 102. doi: 10.4172/2472-1212.1000102

Copyright: $\odot 2015$ Mehta M, et al. This is an open-access article distributed under the terms of the Creative Commons Attribution License, which permits unrestricted use, distribution, and reproduction in any medium, provided the original author and source are credited. 
resuscitation. After the arrest, he required high doses of vasopressors. Out of concern for a new episode of septic shock leading to his arrest, his antibiotics again were broadened to include linezolid and polymyxin $\mathrm{B}$ to cover vancomycin-resistant Enterococcus and multidrug resistant gram-negative bacteria. Polymyxin B was dosed at $300 \mathrm{mg}(3 \mathrm{mg} / \mathrm{kg})$ IV in $100 \mathrm{ml}$ over one-hour followed by $150 \mathrm{mg}(1.5 \mathrm{mg} / \mathrm{kg}) \mathrm{IV}$ over one-hour every 12 hours.

The patient remained afebrile throughout the next several days and cultures remained negative. However, he experienced labile blood pressures, usually requiring little to no vasopressor support but intermittently becoming severely hypotensive requiring high doses of vasopressors. Over the five day period that polymyxin $\mathrm{B}$ was administered, the median SBP/DBP and MAP prior to dosing were 143/58 and 84 (IQR 79-84) and median norepinephrine dose was 1 (IQR 0-2) $\mathrm{microgram} / \mathrm{min}$. After polymyxin $\mathrm{B}$ administration, the median minimum SBP/DBP and MAP fell to 78/36 and 50 (IQR 48-50) and median maximum norepinephrine dose was 8 (IQR 5-21) microgram/ min. The minimum blood pressure after each dose occurred during the infusion or within 30-60 minutes after the infusion ended. The blood pressure consistently returned to baseline within several hours after each dose. In an attempt to mitigate the possible adverse reaction, the infusion was slowed to three hours and administered after a dose of diphenhydramine $25 \mathrm{mg}$. Neither of these strategies mitigated the hypotensive episodes.

On day 15, polymyxin B was discontinued and his blood pressure improved and remained stable off vasopressors for the next eight days. We were unable to obtain a true peak and trough to evaluate his polymyxin B pharmacokinetics. Our patient remained in the medical ICU until hospital day 35 before being discharged to the floor. $\mathrm{He}$ was diagnosed with hemophagocytic lymphohistiocytosis (HLH) and started treatment with dexamethasone and etoposide. His respiratory status improved and he was decannulated from ECMO on day $20 . \mathrm{He}$ later developed hypotension requiring low-dose norepinephrine on days 22 through 26, possibly in the setting of a new infection, but he was not reinitiated on polmyxyin-B. Based on the Naranjo adverse drug reaction score, there appears to be a "probable" relationship between polymyxin $\mathrm{B}$ and hypotension with a score of 5 for both patients.

\section{Discussion}

Due to early findings of nephrotoxicity, polymyxin use was vastly minimized. Thus, key characteristics such as pharmacokinetics and toxicity were not thoroughly elucidated at the time. However, during the last decade, the polymyxins have re-emerged as part of salvage regimens against multidrug resistant gram-negative organisms, prompting new interest in their properties. Although the nephrotoxic and neurotoxic properties are well described in the literature, very few cases describe polymyxin B's cardiovascular toxicity and no other cases describe hypotension.

One possible explanation for hypotension associated with polymyxin $B$ is drug-induced histamine release causing systemic vasodilation and hypotension. Polymyxin-induced histamine release has been shown to correlate with hypotension in rats [1]. Further experiments in cats found that polymyxin B causes rapid and protracted neuromuscular paralysis followed by bradycardia and hypotension, but it is unclear if the cardiovascular and neuromuscular effects are due to the same mechanism [2]. These effects can be prolonged, in particular after repeated exposure to polymyxin $B$. In our first case, the fact that our patient's BP remained low after polymyxin B was discontinued could be consistent with this mechanism. Polymyxin B also has the ability to affect a wide variety of tissues both functionally and morphologically. Based on its activity as a detergent with the ability to disrupt bacterial cell membranes, it has been proposed that polymyxin $B$ also disrupts host cell membranes of many tissues, including the muscle, kidney, and mast cells (among others), thus forming the basis for a unifying theory to explain its various toxicities [2]. Another case report of cardiac arrest after polymyxin B administration also suggests the existence of risk factors that may predispose patients to certain adverse effects with this agent. Dosing, rate of infusion, dilution, addition of a loading dose, and cumulative dose may all have effects on the adverse reaction profile of this agent, but these have not been thoroughly explored [2-4]. Modifying these factors did not seem to be effective in either of our patients.

Our first patient's polymyxin B AUC at 24 hours was $172 \mathrm{mg}$ h/L. This level is much higher than polymyxin B levels observed in other patients at our institution, despite a similar dosing regimen of $3 \mathrm{mg} /$ $\mathrm{kg}$ /day. The abnormally high polymyxin B levels may have contributed to the adverse effects observed. Despite this, we chose not to reduce the dose due to the high polymyxin B MIC (2 mg/L) observed in the E. coli isolate. This decision was based on the results of an in vitro pharmacodynamic study that found the unbound or free AUC (fAUC) to MIC ratio to be the most predictive pharmacokinetic/ pharmacodynamic index correlating to colistin and presumably polymyxin B efficacy [5]. Additionally, a murine thigh model study demonstrated the fAUC/MIC ratio required to achieve a $2-\log 10$ kill was 27.6-36.1 [6]. Assuming a fraction unbound of 0.42 our patient's estimated fAUC was $72 \mathrm{mg}^{\star} \mathrm{h} / \mathrm{L}$. Thus the fAUC/MIC ratio would have been only 36 and dose reductions may have resulted in decreased polymyxin B efficacy.

To our knowledge, there are no other published case reports characterizing polymyxin B-induced hypotension in humans. In the first case described above, we were able to use a relatively high dose for the full course while monitoring blood pressures, while the second patient was not able to tolerate his regimen without developing severe hypotension. Management strategies of slowing the infusion rate and pre-treating with diphenhydramine did not appear to be effective in our patients.

\section{References}

1. Botana LM, Orallo F, Espinosa J, Calleja JM (1986) In vivo inhibition of Polymyxin B-induced hypotension: evidence of beta-adrenergic inhibitory activity on rat mast cells. Gen Pharmacol 17: 615-618.

2. Lee C, Ricker S, Katz RL (1979) Autonomic block, cardiovascular depression, and histamine release produced by polymyxin in the cat. Canad Anaesth Soc J May 26: 196-200.

3. Wunsch H, Moitra VK, Patel M, Dzierba AL (2012) Polymyxin use associated with respiratory arrest. Chest 141: 515-517.

4. Justo JA, Bosso JA (2015) Adverse reactions associated with systemic polymyxin therapy. Pharmacotherapy 35: 28-33.

5. Tam VH, Schilling AN, Vo G, Kabbara S, KwaAL, etal. (2005) Pharmacodynamics of polymyxin B against Pseudomonas aeruginosa. Antimicrob Agents Chemother 49: 3624-3630.

6. Dudhani RV, Turnidge JD, Coulthard K, Milne RW, Rayner CR, et al. (2010) Elucidation of the pharmacokinetic/pharmacodynamic determinant of colistin activity against Pseudomonas aeruginosa in murine thigh and lung infection models 54: 1117-1124. 